\title{
Portable bright-field, fluorescence, and cross-polarized microscope toward point-of-care imaging diagnostics
}

Paul Gordon

Vinicius Paula Venancio

Susanne U. Mertens-Talcott

Gerard Coté 


\title{
Portable bright-field, fluorescence, and cross-polarized microscope toward point-of-care imaging diagnostics
}

\author{
Paul Gordon, ${ }^{a}$ Vinicius Paula Venancio, ${ }^{b}$ Susanne U. Mertens-Talcott, ${ }^{b}$ and Gerard Coté ${ }^{a, c, *}$ \\ ${ }^{a}$ Texas A\&M University, Department of Biomedical Engineering, Optical Biosensing Laboratory, College Station, Texas, United States \\ ${ }^{\mathrm{b}}$ Texas A\&M University, Department of Nutrition and Food Science, College Station, Texas, United States \\ ${ }^{\mathrm{C}}$ Texas A\&M Engineering Experiment Station, Center for Remote Health Technologies and Systems, College Station, Texas, United States
}

\begin{abstract}
Emerging technologies are enabling the feasibility of new types of point-of-care diagnostic devices. A portable, multimodal microscopy platform intended for use in remote diagnostic applications is presented. Use of such a system could bring high-quality microscopy to field use for diseases such as malaria, allowing better diagnostic and surveillance information to be gathered. The microscope was designed using off-the-shelf components and a manual filter selection to generate bright-field, fluorescent, and cross-polarized images of samples mounted to microscopy slides. Design parameters for the system are discussed, and characterization is performed using standardized imaging targets, multimodal phantoms, and blood smears simulating those used in malaria diagnosis. The microscope is shown to be able to image below element 9-3 of a 1951 U.S. Air Force target, indicating that the system is capable of resolving features $<775 \mathrm{~nm}$. Morphological indicators of Plasmodium falciparum can be visualized in images from each modality and combined into high-contrast composite images. To optimize parasitic feature contrast across all three imaging modes, several different staining techniques were compared, with results indicating that use of a single nucleic acid binding fluorophore is preferable. $\odot$ The Authors. Published by SPIE under a Creative Commons Attribution 4.0 Unported License. Distribution or reproduction of this work in whole or in part requires full attribution of the original publication, including its DOI. [DOI: 10.1117/1.JBO.24.9.096502]
\end{abstract}

Keywords: microscope; portable; multimodal; polarized; fluorescence; malaria.

Paper 190128R received Apr. 18, 2019; accepted for publication Sep. 4, 2019; published online Sep. 28, 2019.

\section{Introduction}

Few medical diagnostic tools are as ubiquitously useful as the laboratory microscope. Various tests rely on microscopes to provide information essential to quality medical care around the world. Thus, the lack of microscopy services in many remote areas can be a restriction to the delivery of quality care. Certain diseases, such as mosquito-borne malaria, thrive in such lowresource settings where the lack of access to diagnostic services can stall treatment and eradication of the disease. However, new optical tools may be able to help close the gap by leveraging the ever-improving quality and affordability of sensors and components to build portable, automated diagnostic tools that can be taken to the point of care where high-risk patients reside. This work presents one such system in the form of a portable, digital, and multimodal microscope designed to enable the performance of laboratory-quality imaging for eventual use in the diagnosis of conditions, such as malaria, tuberculosis, sickle-cell anemia, and more in remote locations.

\subsection{Brief History of Portable Microscopy}

Taking microscopy out of a central lab to improve access to patients is not a new proposition. Seminal works by McArthur in the 1930s to 1950 s describe and demonstrate the benefits of point-of-care bright-field microscopy. ${ }^{1-3}$ Since then, numerous iterations of portable microscopes have been proposed and commercialized. $^{4-6}$ A recent, popular approach leverages

*Address all correspondence to Gerard Coté, E-mail: gcote @tamu.edu advances in smartphone technology—network access, processing power, onboard cameras, and other built-in sensors - to construct either intrinsic or extrinsic diagnostic devices. ${ }^{7}$ While intrinsic microscopy systems that are built around cellphones can reduce costs and device complexity, the wide variety of smartphone models and ever-changing camera optics make control of imaging performance difficult. Additionally, most proposed intrinsic smartphone-based microscopes are restricted in their resolution limit due to the low numerical aperture (NA) and high aberration coefficients of the lenses used in their simplified, condensed imaging systems. ${ }^{8}$ Extrinsic devices, such as the one presented here, detect pathologic biomarkers using dedicated external hardware and transmit data to personal electronic devices for review and processing. ${ }^{7}$

\subsection{Innovation}

To broaden the functionality of point-of-care devices toward diagnostic utility and translational potential, this work presents a configuration of a portable microscope capable of gathering images in bright-field, fluorescence, and cross-polarized modes and transmitting the data wirelessly to any web-browser-enabled device for viewing and processing. Numerous portable microscopes have previously reported bimodal functionality, most commonly pairings of either bright-field/dark-field or brightfield/fluorescence imaging. ${ }^{6,9-15}$ However, combinations of three or more imaging modalities have been almost exclusively relegated to sophisticated benchtop instruments. ${ }^{16-21}$ On the benchtop, combinations of bright-field, fluorescence, and crosspolarized imaging have been validated, yet never translated into 
portable configurations for use at the point of care. ${ }^{22,23}$ This system, designed using off-the-shelf optical components, is easily adaptable to the needs of a variety of imaging tasks, making it, to the authors' knowledge, the first published example of a portable, customizable, and trimodal microscopy platform.

\subsection{Application and Potential Impact}

Microscopy for malaria diagnosis was selected as the initial target application for the microscope for its high potential impact, established imaging standards, and demonstrated diagnostic utility for each individual imaging modality. ${ }^{24-35}$ Indeed, it is hoped that disclosure of this tool may facilitate investigations of an integrated trimodal microscopy approach to malaria diagnosis. Currently, the gold-standard test to diagnose malaria uses $1000 \times$ magnification benchtop bright-field microscopy to examine thick and thin Giemsa-stained peripheral blood smears to identify and quantify the presence of Plasmodium parasites. ${ }^{24}$ This test requires a well-equipped and well-staffed microscopy lab, which is often unavailable in remote regions where the disease is most common. Where access to a centralized lab is restricted, diagnoses are frequently made using only symptoms, substandard microscopy, or rapid diagnostic tests, which can lead to overdiagnosis and misdiagnoses. ${ }^{36-38}$ Frequently, antimalarial medications are prescribed to patients without a confirmed diagnosis, which can waste resources, cause other diseases to go untreated, potentially lead to ineffective treatment, and increase risks for developing partial drug resistance. $^{39-41}$

\section{Materials and Methods}

\subsection{Optical System Design Considerations}

Because microscopic diagnosis of blood-borne pathogens such as Plasmodium relies on the identification of morphological characteristics, the microscopy system must achieve a theoretical diffraction-limited resolution smaller than the sought-after features. For this system, the resolution target was set at $0.5 \mu \mathrm{m}$ in order to resolve chromatin dots and gross features of ring-stage infections that average between 0.5 and $1 \mu \mathrm{m}$ in size. ${ }^{42}$ This limit then drives the NA of the objective and condenser lenses in the system according to the Rayleigh criterion as described by

$$
d_{x, y}=\frac{1.22 \lambda}{\left(\mathrm{NA}_{\mathrm{obj}}+\mathrm{NA}_{\mathrm{cond}}\right)},
$$

where $d_{x, y}$ is the minimum laterally resolvable distance between two points; $\lambda$ is the wavelength of light; and $\mathrm{NA}_{\text {obj }}$ and $\mathrm{NA}_{\text {cond }}$ are the objective and condenser NA, respectively. For this work, an Olympus MPlan 50×, $0.75 \mathrm{NA}$, infinity corrected objective was selected for use, and, because the condenser NA is $\geq 0.75$, it is theoretically capable of resolving points separated spatially by $423 \mathrm{~nm}$ at wavelengths of $520 \mathrm{~nm}$ according to Eq. (1). Any infinity corrected microscope objective may be used in the system to optimize magnification, field of view, and imaging performance according to the needs of a specific test, although users should be aware that performance may not remain consistent across objectives from various manufacturers, as manufacturer-specific tube lenses are not used.

\subsection{Optical System Configuration}

The overall microscope configuration is depicted in Fig. 1 and was previously reported as a proceeding of SPIE. ${ }^{43}$ Both epi and transmission illumination are available, depending on the desired imaging mode. A 3D-printed housing holds elements of the simplified Köhler transmission illumination system, in which light from a warm-white light-emitting diode (LED) (XREWHT-L1-R250-009F7, Cree) is collected by a singlet asphere (ACL25416U-A, Thorlabs), transferred through a field diaphragm, and, after being folded $90 \mathrm{deg}$ by a plane mirror (PFSQ10-03-P01, Thorlabs), is passed through a linear polarizer sheet (PS030-R15, MidOpt) and condenser aperture before being focused onto the sample plane by a second identical asphere, which has an NA of 0.79 . In epi-illumination mode, such as for fluorescent imaging, light from a blue, 485-nm LED (XPEBBL-L1-0000-00301, Cree) is collected by a similar singlet asphere, passed through an excitation filter (\#67-028, Edmund Optics), reflected off of a dichroic mirror (\#67-080, Edmund Optics), and condensed onto the sample plane through the microscope objective. Both LEDs are driven at 3-V forward voltage, resulting in expected luminous flux output of 80 lumen from the white and 45 lumen from the blue. From the sample, image-encoded light is collected by the objective, passed through the dichroic mirror and one of three available filters in infinity space, then focused onto the camera by a tube lens with 100-mm focal length (TTL100-A, Thorlabs). A third-party, reduced focal length tube lens was chosen to reduce optical system path length and because such a lens is more likely to be useful with objectives from a variety of manufacturers. Both the imaging and transmission illumination paths are folded using planar mirrors to reduce the overall footprint of the system.

\subsection{Generation of Multimodal Contrast}

In this configuration, the microscope can gather images in different contrast modes without moving the sample focus or alignment. To select which mode of imaging is used, the only changes necessary are to activate the appropriate LED source and select the proper filter from the sliding filter deck. To generate bright-field images, the transmission illumination, warmwhite LED is activated and a blank space in the filter deck is selected. The polarizing filter and dichroic mirror are by default kept in the optical path, which prefilters all light reaching the sample in bright-field imaging to be linearly polarized and filters all light reaching the camera to be of wavelengths longer than the dichroic cutoff. This places an inherent limitation on the amount of chromatic information available in bright-field imaging but can be compensated using simple postprocessing to recreate images in a traditional color-space by interpolating information from missing color channels. Alternatively, if the information contained in wavelengths shorter than the dichroic cutoff is critical for a particular test, the dichroic filter can easily be removed from the system but must be replaced prior to collection of fluorescent images.

In fluorescence imaging mode, a board-mounted blue monochromatic 485-nm epi-LED is activated and fluorescent emission filter (\#67-031, Edmund Optics) selected from the filter deck. These excitation and emission bands were selected because most prevalent fluorophores used in malaria diagnosis utilize fluorescein isothiocyanate (FITC) excitation/emission wavelengths with cut on/off at $500 \mathrm{~nm}$, although any visible excitation and emission bands may be used. ${ }^{35}$ For cross-polarized 


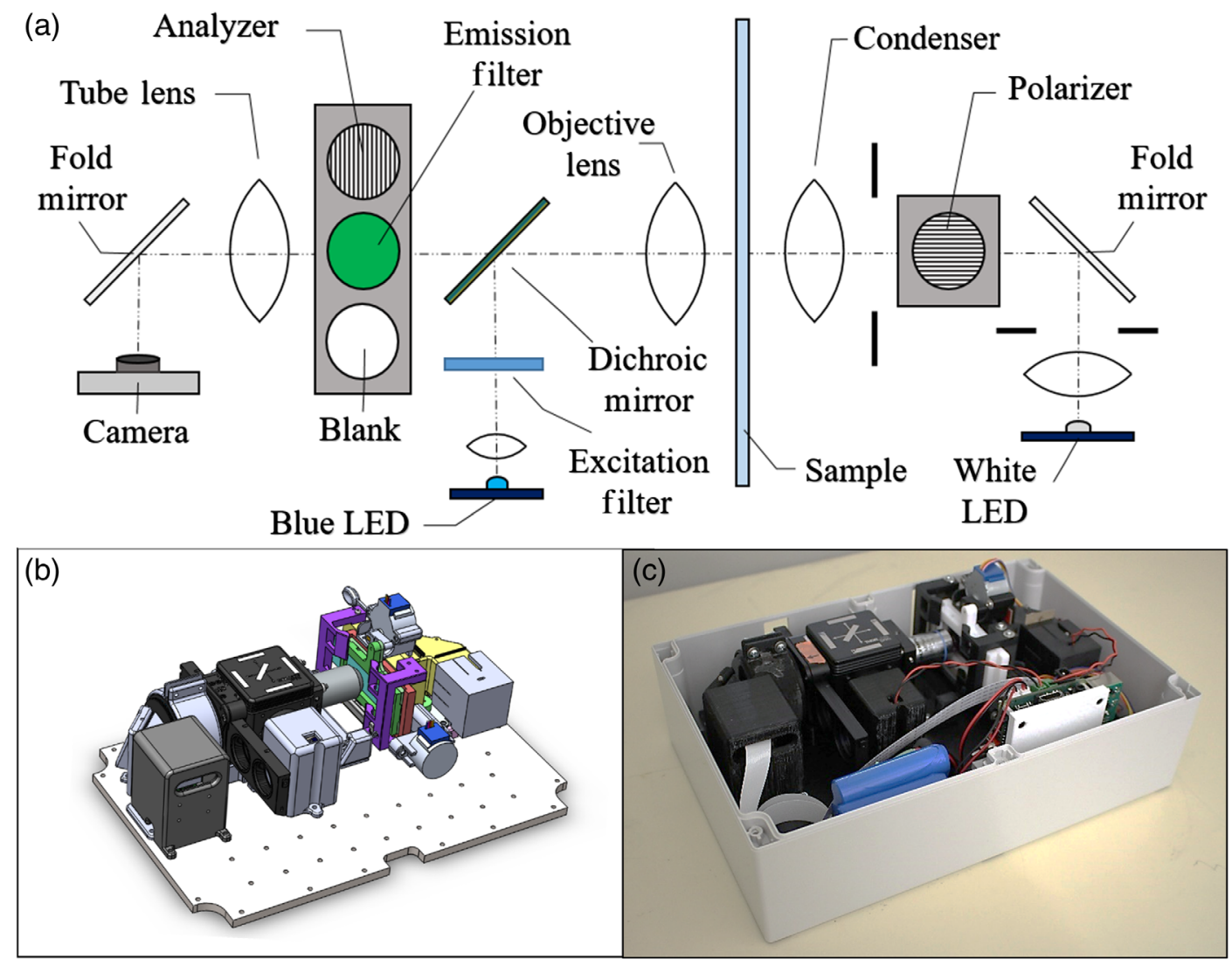

Fig. 1 Diagram of portable microscope system layout and completed prototype microscope: (a) system layout diagram, (b) system CAD model, and (c) final microscope system prototype.

imaging, the transmission LED is activated and analyzing filter selected from the deck. When transmission illumination is used, all light passing through the system is also linearly polarized by default, as the polarizing filter is permanently kept in the Köhler illumination system. It was discovered, both in the literature and experimentally, that the dichroic mirror will reduce the crosspolarized signal intensity unless the polarizer and analyzer are carefully aligned with the orthogonal $X$ and $Y$ axes of the dichroic mirror to minimize circular or elliptical polarization states generated at the mirror. $^{44,45}$ Alignment of the polarization to within $0.5 \mathrm{deg}$ of orthogonal with the reflection plane of the dichroic was found to be required to generate an adequate extinction ratio, which allows for the imaging of depolarizing biomarkers in samples.

\subsection{Electronic and Optomechanical Components}

A Raspberry Pi 3 was selected as the data collection, storage, and transmission module for the microscope due to its low cost, ease in prototyping, and simple integration with commercially available camera sensors. A complementary 8 MP Picam v.2 with Sony IMX219PQ sensor $(3.674 \times 2.760 \mathrm{~mm}, 1.12-\mu \mathrm{m}$ pitch) with lens removed was selected for its small pixel size, resolution, low cost, and highly controllable acquisition parameters. To control sample translation and focusing, four mini stepper motors (28BYJ-48, Kiatronics, New Zealand) with 4096 step increments/rotation receive driving currents from a custom PCB that translates commands from the Raspberry Pi into discreet step intervals. Two motors control focal depth, one controls $X$ axis sample translation and the other controls $Y$ axis translation. Power is provided to the microscope by two lithium-ion battery packs that can be recharged by plugging the microscope into a power outlet. The optomechanical mounts for the prototype microscope were either $3 \mathrm{D}$ printed or incorporated as off-the-shelf parts.

\subsection{Operational Procedure}

To operate the microscope, a thin sample fixed to a standard $25 \times 75 \mathrm{~mm}$ microscope slide is inserted into the stage via access port opened from the side of the case. Wet samples with coverslips may also be used, although it is recommended to affix the coverslip to the slide to prevent slippage while in the stage. The microscope is powered on, and any portable device with web-interface can be connected to the microscope via a device generated Wi-Fi network. After connection, a secure shell (SSH) portal interface can be used to access controls for sample translation, focal control, and imaging mode. Adjustments to the sample position or focus can be made if necessary, or an alternative imaging modality can be selected without altering the sample position. A new field of view may be found using the interface to move to any immediately adjacent field of view using preprogrammed commands. After pictures are captured, they can be automatically uploaded to any database structure desired, either via remote network access, to onboard memory, or directly to the user's personal device. To demonstrate the capability of the multimodal microscope to interface with personal electronic devices, images were captured using a Windows-based laptop PC, an Android-based smartphone, and an Apple iPad (Fig. 2). On all platforms, the command interface was able to successfully control microscope function and collect multimodal data. 

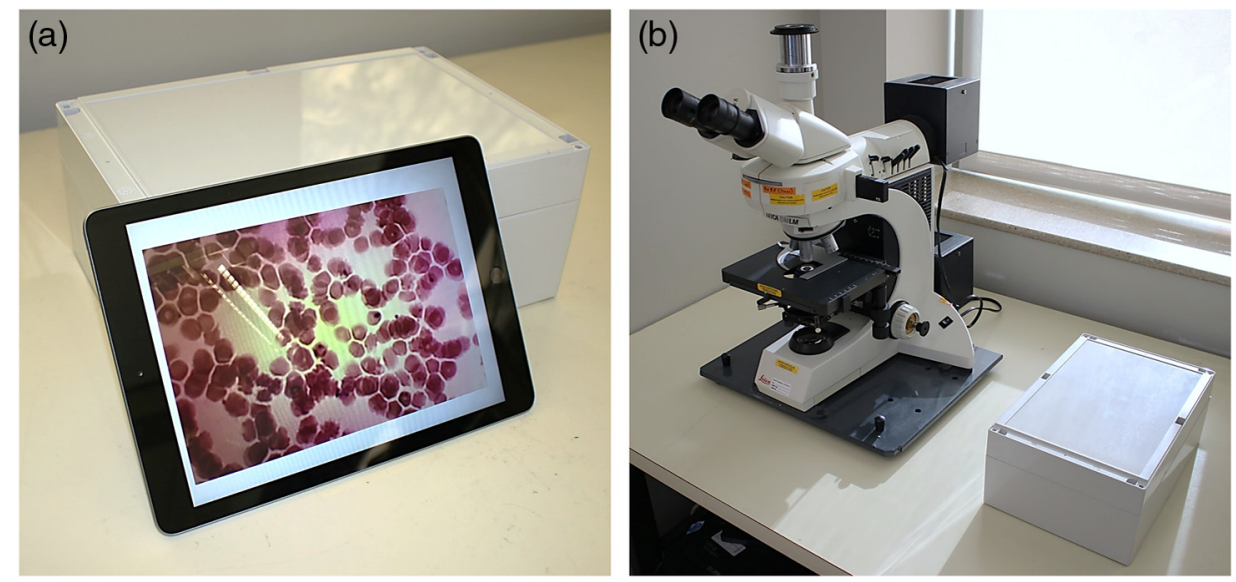

Fig. 2 Image of the final portable system (a) in use with an iPad and (b) next to a comparable benchtop instrument.

\subsection{Sample Preparation}

To assess the multimodal functionality of the portable system, a control phantom was created by fluorescently staining lanuginose fibers from the underside of a sericeous tree leaf using SYBR-1 Green nucleic acid stain (Sigma) for $20 \mathrm{~min}$, then embedding the fibers in a thin film of degassed polydimethylsiloxane (Sylgard 184) on the surface of a microscope slide. A Zeiss AxioVert-A1 fluorescence microscope with high-power broadband LED source and FITC filter cube was used as the fluorescence control, and a Leica DMLM compound microscope was used for both bright-field and cross-polarized controls. The embedded fibers express absorption in white light, birefringence under cross-polarization, and strong fluorescence at the excitation and emission wavelengths of the portable system. To demonstrate the ability of the microscope to be used in diagnostic applications, blood smears of in vitro cultured Plasmodium falciparum were stained with either SYBR Green1 dye or a dual Giemsa/fluorophore technique described by Guy et al. ${ }^{32}$ and imaged using the microscopy system. The two staining methods were compared as possible sample preparation options to ascertain which would provide the optimal balance of biomarker contrast across all three contrast modes, which has not been previously addressed in the literature.

\section{Experimental Results}

\subsection{Performance Characterization}

To assess system resolution capabilities, a negative high-resolution 1951 U.S. Air Force target (transmissive line elements) was first used to test the resolving capabilities and magnification of the system. As can be seen in Fig. 3, element 9-3 (775-nm linewidth) of the target is fully resolved using full-width-half-max criteria, easily satisfying both Rayleigh's and Sparrow's criteria for these features. Using known linewidths and camera pixel pitch, images of the U.S. Air Force target were used to experimentally calculate the net optical system magnification to be $27.8 \times$. Images of the multimodal control phantom used to assess multimodal function are shown in Fig. 4, with the same field of view imaged both on the portable microscope and gold-standard benchtop systems.

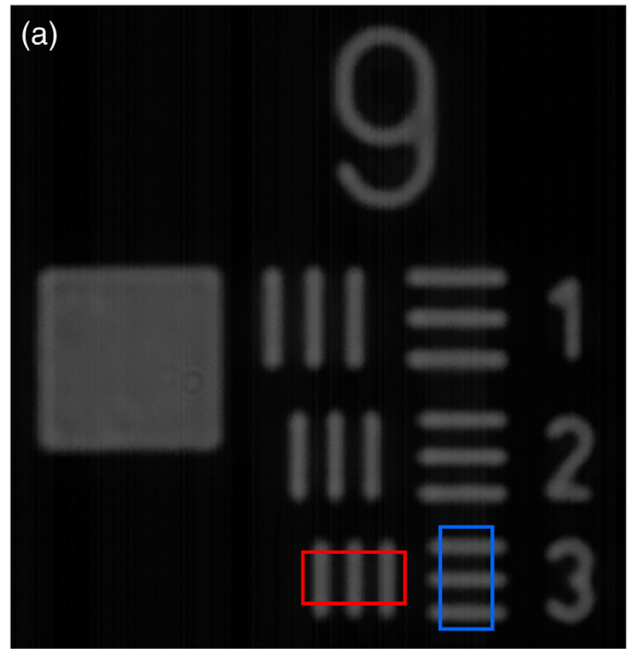

(b)

Air-force target sections

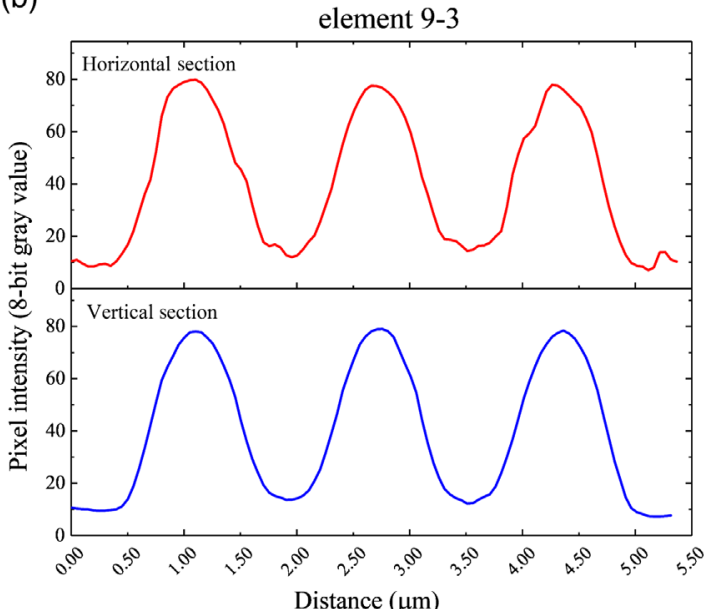

Fig. 3 Resolution capability of the system as shown by (a) imaged 1951 U.S. Air Force target. (b) Line contrasts were averaged over the vertical and horizontal elements using boxed regions as shown using ImageJ software. 

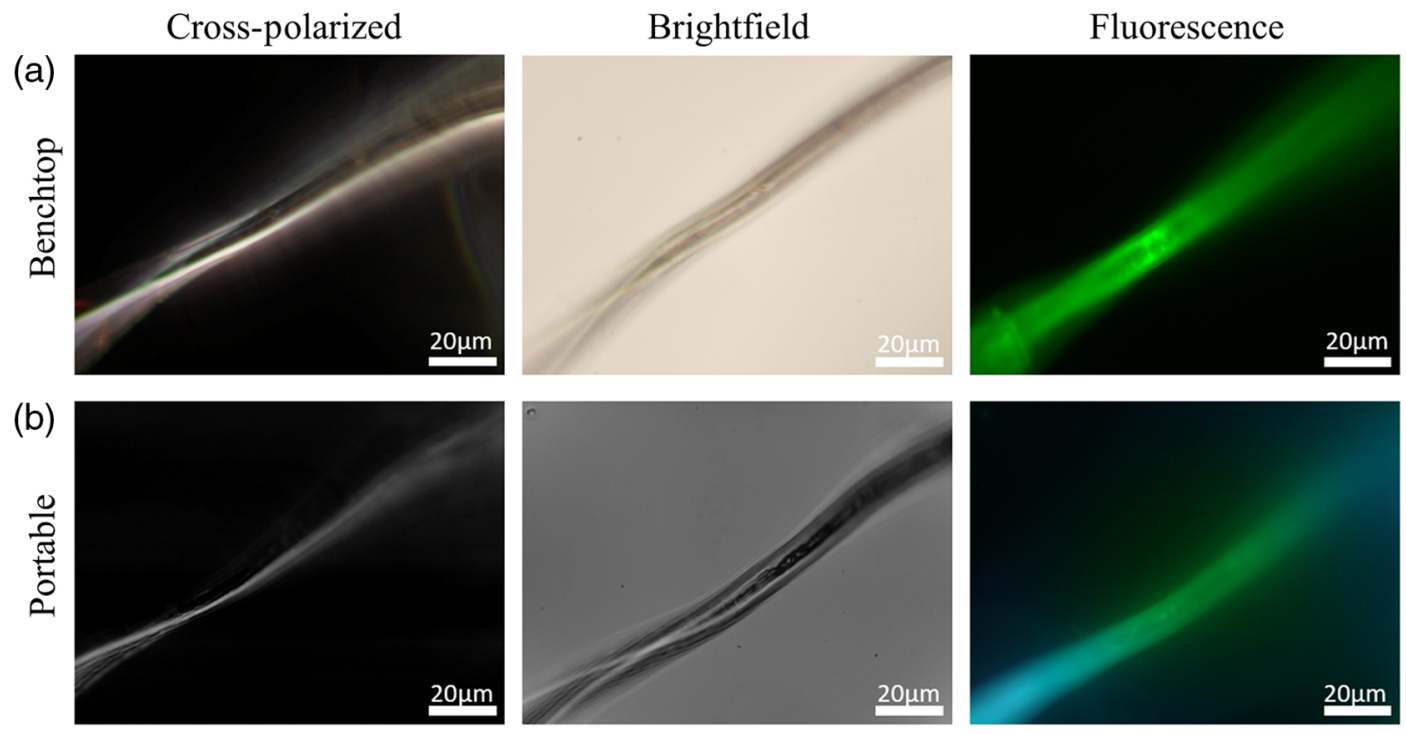

Fig. 4 Images of a multimodal phantom collected on (a) individual benchtop instruments and (b) the portable microscopy system. The same field of view is imaged on each system.

\subsection{Plasmodium Sample Preparation Optimization}

Thin films of blood cultured with P. falciparum were stained using either dual-staining or SYBR Green-1 methods as previously referenced, and images of these smears collected using the portable microscope were analyzed to assess signal strengths for each preparation protocol across each mode. Figure 5 shows the average pixel intensity of various parasitic morphological features from grayscale images of this comparative study. Sample variance was defined as the standard deviation of the population of signal values surveyed. Bright-field images clearly show cell membranes and intracellular malaria pigment using either staining technique. Dual-stained images express a slightly darker background than those stained with SYBR Green-1 alone; however, no significant difference was found for the absorption of light in trophozoites between the two staining methods [Fig. 5(a)]. Fluorescence images reveal no significant difference in background intensity between the two staining techniques, although samples stained only with SYBR Green-1 showed significantly higher signal in trophozoites and schizonts, which is desirable for signal collection efficiency with low-cost photodetector arrays [Fig. 5(b)].

In cross-polarized images, the signal strength from light depolarization in birefringent hemozoin crystals was found to be higher on average for SYBR-stained samples than for those of dual-stained images [Fig. 5(c)]. Lower average cross-polarized signals in dual-stained samples were not expected, although the occurrence of these phenomena may be explained by the fact that Giemsa stain, which is part of the dual-staining technique, frequently precipitates salt crystals from its ionic components, which would add to the total number of depolarization events detected in the dual-stained sample and lower the average signal intensity if said precipitate crystals express weaker retardation than hemozoin. This effect, in combination with higher fluorescence signal intensities, implies that use of SYBR Green-1 or other comparable fluorophores appears to be a logical choice for maximizing the amount of useful information that can be gathered from the portable multimodal microscopy system, although the specific preparation techniques must necessarily vary depending on the specific sample and test to be performed. ${ }^{46}$

\subsection{Multimodal Plasmodium falciparum Imaging}

Thin smears of $P$. falciparum stained with SYBR Green-1 were imaged using the portable microscope and a benchtop Nikon Eclipse inverted microscope as a gold-standard control. Images from all three modalities of the portable system were successfully collected in succession and wirelessly transmitted to
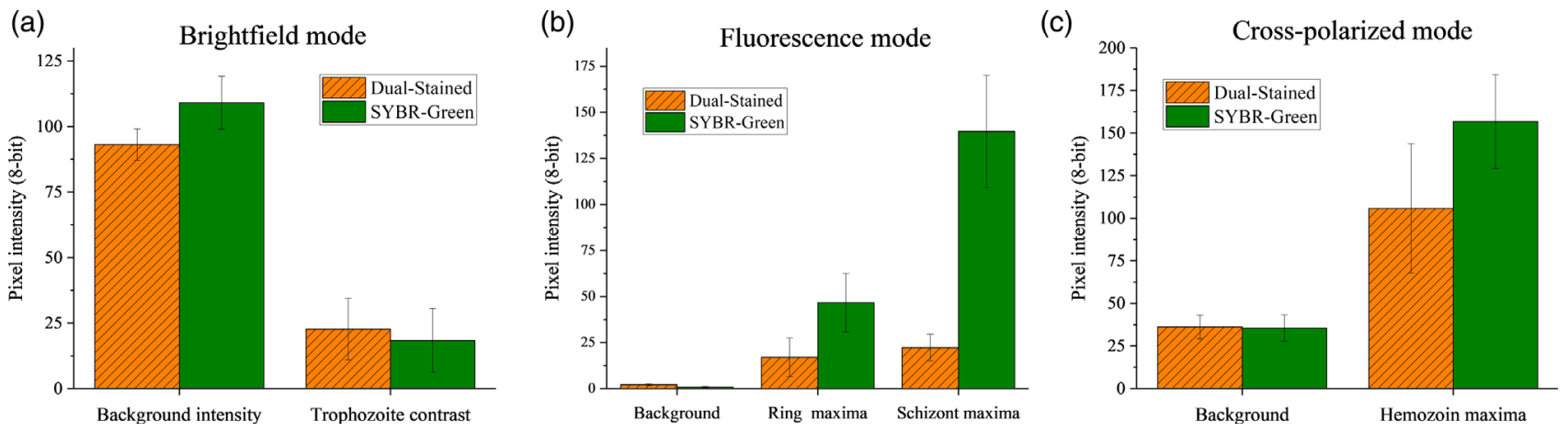

Fig. 5 Metrics from sample preparation tests to optimize multimodal information available from $P$. falciparum cultured blood smears in (a) bright-field, (b) fluorescence, and (c) cross-polarized images. 
a personal electronic device for analysis. The results of multimodal imaging shown in Fig. 6 show that the portable instrument is capable of producing images of comparable image quality to benchtop instruments for each contrast modality. Images taken using the portable system were previously reported in an SPIE proceeding. ${ }^{47}$ Diagnostically relevant morphological features were able to be observed in images from all three modes on both systems. All images were converted to grayscale during analysis. This was done primarily for aesthetic purposes, as it minimizes confusion resulting from chromatic shifts that appear when bluechannel data are removed from the bright-field color images, and fluorescence and cross-polarized images could then be false colored to heighten contrast in the displayed images.

Portable bright-field images clearly show cell boundaries and intracellular parasites, although they do not display the same level of contrast between the intracellular space and background as is seen on benchtop images. In fluorescent images, the portable system is able to clearly resolve dual chromatin dots in ringstage trophozoites, which are characteristic of $P$. falciparum infections. In cross-polarized mode, the portable system reveals the presence of hemozoin within a schizont; however, it was unable to detect as many crystals as the benchtop microscope, indicating that the portable system suffers from a loss of contrast in this mode. In general, it was found that the system is able to resolve intra-erythrocytic parasites in all three imaging modalities, though with decreased contrast ratios when compared to benchtop systems using similar magnification. The portable multimodal images show various small parasitic features such as intracellular vacuoles, chromatin dots, ring stage and mature trophozoites, schizonts, and large hemozoin crystals.

From images collected on both the portable and gold-standard systems, image quality metrics and parasitic counts were collected to quantify the performance of the portable system. Figure 7 presents bar graphs showing the mean intensity of various morphological features in images of each modality, with data normalized to the mean of the feature with highest intensity in each to compensate for differences in exposure intensities and times between the portable and benchtop systems. Between 3000 and 3500 cells were surveyed using each system. For parasitemia analysis, the infected proportion of cells was treated as a Bernoulli distribution, with binary determination of either "infected" or "uninfected" made for each cell and variance expressed as the binomial proportion confidence interval.

Both systems present general agreement of feature signal contrast across all three modes, and measured parasitemia generally agreed with the nominal value of $5 \%$ of cells containing parasites. In bright-field images normalized to the background intensity, the portable system displays similar values but higher variance than the benchtop control for background and intracellular regions, which is expected given the lower quality
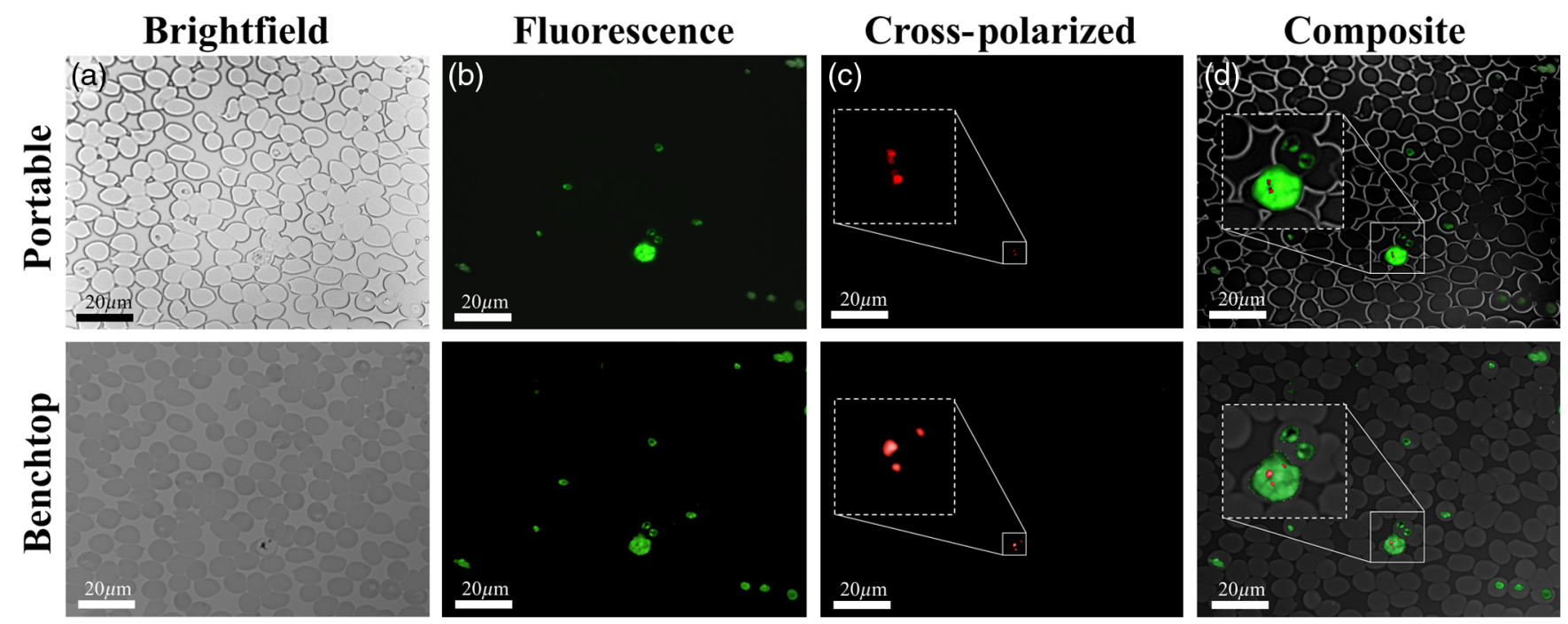

Fig. 6 Multimodal images of various Plasmodium morphological forms from a single field of view: (a) bright-field images, (b) fluorescence images, (c) cross-polarized images, and (d) composite images of all three modes.

(a)

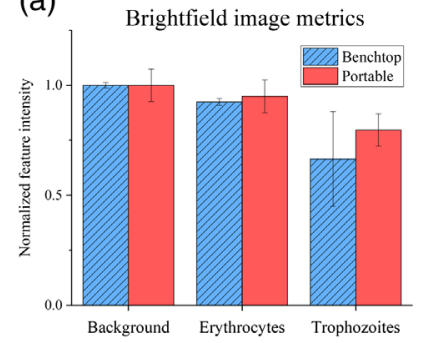

(b)

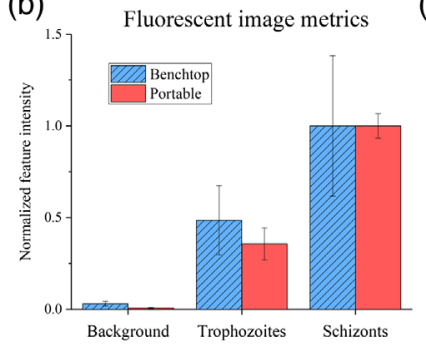

(c)

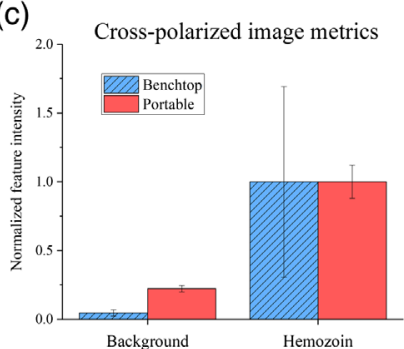

(d)

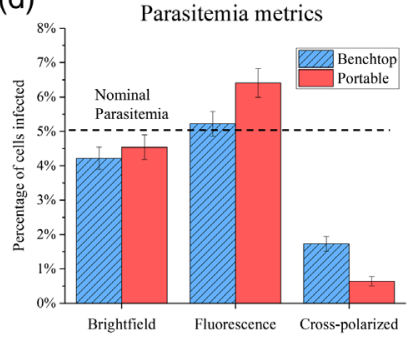

Fig. 7 Comparative metrics for images of the same sample taken on both portable and benchtop microscopes. Common diagnostic biomarkers are shown for (a) bright-field, (b) fluorescence, and (c) cross-polarized images, with (d) measurements of quantitative parasitemia shown for each model. 
of camera and optical components utilized. However, this trend is reversed when trophozoites are examined, with the benchtop images displaying significantly higher variance of contrast compared to the portable system. This trend will be repeated across images from all three modes, with the portable system displaying higher signal variance for homogenous image regions such as the background or intracellular spaces, whereas benchtop images reveal higher signal variance for heterogeneous features. It is believed that this occurs because the benchtop system provides greater modulation of the high spatial frequencies found in fine, heterogeneous features, allowing the full range of feature intensities to be captured. In contrast, while the optical system of the portable microscope can resolve such small features, it does not modulate the high spatial frequencies as effectively, leading to a loss in the dynamic range of signals. Images of trophozoites and schizonts in fluorescence images display this pattern as well. It must be noted, however, that fluorescence parasitemia data show that the portable system may be registering false positives in $\sim 1.5 \%$ of cells, meaning that artifact registration may skew the feature intensity averages presented in Fig. 7(b). Hemozoin signals from benchtop cross-polarized images likewise show significantly higher variance, with the cause attributed also to the higher number of small crystals that are able to be seen above the image noise floor, which is supported by analysis of the number of cells observed to contain the biomarkers in Fig. 7(d).

\section{Discussion}

\subsection{Interpretation of Results}

The portable microscope is able to collect images in each modality with feature resolution below $775 \mathrm{~nm}$, which is sufficient to detect biomarkers for many cellular samples. Indeed, examination of composite images collected from P. falciparum samples clearly show morphological features necessary to detect infections, quantify parasitemia, and distinguish mature parasites containing hemozoin, all of which are diagnostically important metrics currently not available from a single standard point-ofcare device. Morphological feature recognition was performed by authors with several years of experience examining malaria smears in a research laboratory environment. This imparts subjective bias into the process of recognizing and cataloging morphological features in images, and further analysis by either independent trained experts in malaria hematology or by automated feature recognition software is necessary to improve the level of absolute confidence in the ability of the microscope to resolve parasitic morphology.

Limitations of system optics must be acknowledged, including the loss of some chromatic information in bright-field images due to inclusion of the dichroic mirror, reduced contrast in high spatial frequency information, increased exposure times necessary for fluorescence images due to use of a noncooled, color camera sensor, and reduced extinction ratios in cross-polarized mode when compared to a gold-standard benchtop instrument. Additionally, the presence of apparent false positives in fluorescence images is yet uncharacterized and must be studied further, after which it is hoped that image processing techniques may be able to separate artifacts from the true positives in datasets. Despite these limitations, the system represents an advancement in the quality and flexibility of microscopy systems designed for use at the point of care and may certainly have applications beyond those described in this work.

\subsection{Device Usability}

All components of the multimodal imaging system are self-contained in a durable plastic enclosure measuring $11^{\prime \prime} \times 6^{\prime \prime} \times 5^{\prime \prime}$, with auxiliary components such as the Raspberry Pi, motor control board, and rechargeable battery packs fully integrated into the unit. The unit is capable of operating without any external power supply from onboard battery packs or may be plugged into an external $5-\mathrm{V}$ power supply for operation and battery recharge. An advantage of the system is its flexibility to be easily modified to match the specific needs of a variety of specific tests that may be required. For example, if images of a different magnification or fluorescence emission spectrum are desired, components such as the objective lens, emission filters, LEDs, and dichroic mirror are easily accessible and can be interchanged to adapt the microscope to achieve the desired performance. This ability to customize the microscope underscores its potential utility as a platform for performing laboratory-grade microscopic imaging at the point of care for a variety of applications. The system as presented here is not without its usability limitations, as numerous changes would likely be made to translate the current prototype into a commercially viable unit that is low cost and field-ready. Use of off-the-shelf or 3D-printed optomechanical components should be replaced by inexpensive, appropriately toleranced injection-molded parts to improve repeatability and reduce costs. Additionally, the water, shock, and dust resistance and overall durability have not yet been fully tested for the prototype and would need to be considered before field use.

\section{Conclusion}

In conclusion, we have shown that a portable, multimodal microscope can successfully resolve features necessary for detecting P. falciparum parasites in bright-field, fluorescence, and cross-polarized modalities. This system is constructed in a compact form factor using standard optical components and inexpensive electronics. Preliminary results toward use for malaria diagnosis show that the same morphological features are able to be seen on the portable system as on a benchtop microscope with similar magnification, indicating that further, more comprehensive investigation is merited to assess its sensitivity and specificity in a comprehensive diagnostic setting. To the authors' knowledge, this is the first published example of a portable, customizable, trimodal microscope intended for diagnostic applications at the point of care.

\section{Disclosures}

The authors of this paper declare no conflicts of interest relevant to the claims set forth herein.

\section{Acknowledgments}

The authors wish to thank Masih Ghorayshi for his assistance in preparing and staining blood smears for analysis for this study; Richard Horner and Cody Lewis for their design and assembly of the electronic systems used for sample control; and Kevin Kiefer and Taila Kawano for their assistance with growing parasitic cultures and creating blood smears used for analysis. This work was made possible by contributions from the National Science Foundation (NSF) in the form of Research Grant No. 1402846, graduate research fellowship positions (GRFP \#2017240275), and the Precise Advanced Technologies and Health Systems for Underserved Populations (PATHS-UP) Engineering Research Center (1648451). 


\section{References}

1. J. McArthur, "II.-Advances in the design of the inverted prismatic microscope," J. R. Microsc. Soc. 65(1-4), 8-16 (1945).

2. J. McArthur, "A new concept in microscope design for tropical medicine," Am. J. Trop. Med. Hyg. 7(4), 382-385 (1958).

3. J. N. McArthur, "XIII.-A new type of portable microscope," $J$. R. Microsc. Soc. 54(3), 182-185 (1934).

4. J. R. Richards, K. A. Gaylor, and A. J. Pilgrim, "Comparison of traditional otoscope to iPhone otoscope in the pediatric ED," Am. J. Emergency Med. 33(8), 1089-1092 (2015).

5. K. Dunning and J. R. Stothard, "From the McArthur to the millennium health microscope (MHM): future developments in microscope miniaturization for international health," Microsc. Today 15(2), 18-21 (2007).

6. D. Jones et al., "McArthur revisited: fluorescence microscopes for field diagnostics," Trends Parasitol. 23(10), 468-469 (2007).

7. L. Kwon et al., "Medical diagnostics with mobile devices: comparison of intrinsic and extrinsic sensing," Biotechnol. Adv. 34(3), 291-304 (2016).

8. N. A. Switz, M. V. D’Ambrosio, and D. A. Fletcher, "Low-cost mobile phone microscopy with a reversed mobile phone camera lens," PLOS One 9(5), e95330 (2014).

9. K. K. Ghosh et al., "Miniaturized integration of a fluorescence microscope," Nat. Methods 8(10), 871-878 (2011).

10. A. R. Miller et al., "Portable, battery-operated, low-cost, bright field and fluorescence microscope," PLoS One 5(8), e11890 (2010).

11. S. Schaefer, S. A. Boehm, and K. J. Chau, "Automated, portable, lowcost bright-field and fluorescence microscope with autofocus and autoscanning capabilities," Appl. Opt. 51(14), 2581-2588 (2012).

12. Y. S. Zhang et al., "A cost-effective fluorescence mini-microscope for biomedical applications," Lab Chip 15(18), 3661-3669 (2015).

13. D. N. Breslauer et al., "Mobile phone based clinical microscopy for global health applications," PLoS One 4(7), e6320 (2009).

14. C. W. Pirnstill and G. L. Coté, "Malaria diagnosis using a mobile phone polarized microscope," Sci. Rep. 5, 13368 (2015).

15. Q. Wei et al., "Plasmonics enhanced smartphone fluorescence microscopy," Sci. Rep. 7(1), 2124 (2017).

16. M. Brydegaard et al., "Versatile multispectral microscope based on light emitting diodes," Rev. Sci. Instrum. 82(12), 123106 (2011).

17. S. G. Demos et al., "Imaging of tissue microstructures using a multimodal microscope design," IEEE J. Sel. Top. Quantum Electron. 11(4), 752-758 (2005).

18. E. Salmon et al., "High resolution multimode digital imaging system for mitosis studies in vivo and in vitro," Biol. Bull. 187(2), 231-232 (1994).

19. T. Nagano et al., "Incident-light fluorescence microscope," U.S. Patent No. 6,025,956 (2000).

20. C. Li and Y. Zhu, "Dual function microscope for quantitative DIC and birefringence imaging," Proc. SPIE 9713, 97131I (2016).

21. R. Patel et al., "Multimodal optical imaging for detecting breast cancer," J. Biomed. Opt. 17(6), 066008 (2012).

22. Y. Liu et al., "Complementary fluorescence-polarization microscopy using division-of-focal-plane polarization imaging sensor," J. Biomed. Opt. 17(11), 116001 (2012).

23. A. N. Yaroslavsky and R. R. Anderson, "Fluorescence polarization imaging devices and methods," Google Patents (2007).

24. World Health Organization, Basic Malaria Microscopy, WHO, Geneva (2010).

25. World Health Organization, Malaria Fact Sheet, WHO, Geneva (2017).

26. M. P. Grobusch et al., "Sensitivity of hemozoin detection by automated flow cytometry in non-and semi-immune malaria patients," Cytometry, Part B 55(1), 46-51 (2003).

27. T. Hänscheid, "The future looks bright: low-cost fluorescent microscopes for detection of mycobacterium tuberculosis and coccidiae," Trans. R. Soc. Trop. Med. Hyg. 102(6), 520-521 (2008).

28. T. Hänscheid, "Diagnosis of malaria: a review of alternatives to conventional microscopy," Clin. Lab. Haematol. 21(4), 235-245 (1999).

29. T. Hänscheid et al., "Full blood count and haemozoin-containing leukocytes in children with malaria: diagnostic value and association with disease severity," Malaria J. 7(1), 109 (2008).
30. T. Hänscheid, E. Valadas, and M. Grobusch, "Automated malaria diagnosis using pigment detection," Parasitol. Today 16(12), 549-551 (2000).

31. M. Rebelo et al., "Haemozoin detection in infected erythrocytes for Plasmodium falciparum malaria diagnosis-Prospects and limitations," Acta Trop. 123(1), 58-61 (2012).

32. R. Guy et al., "The use of fluorescence enhancement to improve the microscopic diagnosis of falciparum malaria," Malaria J. 6(1), 89 (2007).

33. L. Gwanzura et al., "Evaluation of 'cyscope', a novel fluorescencebased microscopy technique for the detection of malaria," J. Infect. Dev. Countries 6(02), 212-215 (2011).

34. F. Kawamoto, "Rapid diagnosis of malaria by fluorescence microscopy with light microscope and interference filter," Lancet 337(8735), 200-202 (1991).

35. B. Lowe et al., "Acridine orange fluorescence techniques as alternatives to traditional giemsa staining for the diagnosis of malaria in developing countries," Trans. R. Soc. Trop. Med. Hyg. 90(1), 34-36 (1996).

36. World Health Organization, "Malaria rapid diagnostic test performance: results of WHO product testing of malaria RDTs: round 6 (2014-2015)" (2015).

37. R. E. Coleman et al., "Comparison of field and expert laboratory microscopy for active surveillance for asymptomatic Plasmodium falciparum and Plasmodium vivax in western Thailand," Am. J. Trop. Med. Hyg. 67, 141-144 (2002)

38. C. Drakeley and H. Reyburn, "Out with the old, in with the new: the utility of rapid diagnostic tests for malaria diagnosis in Africa," Trans. R. Soc. Trop. Med. Hyg. 103(4), 333-337 (2009).

39. B. Hanboonkunupakarn and N. J. White, "The threat of antimalarial drug resistance," Trop. Dis. Travel Med. Vaccines 2(1), 10 (2016).

40. O. O. Oladosu and W. A. Oyibo, "Overdiagnosis and overtreatment of malaria in children that presented with fever in Lagos, Nigeria," ISRN Infect. Dis. 2013, 1-6 (2012).

41. W. P. O'Meara, D. L. Smith, and F. E. McKenzie, "Potential impact of intermittent preventive treatment (IPT) on spread of drug-resistant malaria," PLoS Med. 3(5), e141 (2006).

42. G. S. Noland, N. Briones, and D. J. Sullivan, "The shape and size of hemozoin crystals distinguishes diverse plasmodium species," Mol. Biochem. Parasitol. 130(2), 91-99 (2003).

43. P. Gordon et al., "A portable microscopy system for fluorescence, polarized, and brightfield imaging," Proc. SPIE 10485, 104850N (2018).

44. E. Hecht, Optics, 4th ed., Addison-Wesley, San Francisco (2002).

45. T. Erdogan, "Optical filters polarization and filters," Presentation File, Semrock Corporation, semrock.com (2011).

46. R. W. Horobin and J. A. KiernanConn's Biological Stains, 10th ed., BIOS Scientific Publishers Ltd., Oxford (2002).

47. P. Gordon et al., "Diagnostic utility of a portable multimodal microscope for malaria treatment at the point-of-care," Proc. SPIE 10869, 108690X (2019).

Paul Gordon is a PhD candidate at Texas A\&M University in the Department of Biomedical Engineering. He received his BS degree in biomedical engineering from the University of Cincinnati in 2015. His current research interests include various point-of-care optical technologies including portable microscopy, on-chip spectroscopy, and innovative fabrication techniques for low-cost optical components. $\mathrm{He}$ is a student member of SPIE, NSF Graduate Research Fellow, and a student fellow of the PATHS-UP Engineering Research Center.

Gerard Coté is a Texas A\&M University Regents Professor and the James J. Cain Professor I in biomedical engineering at Texas A\&M University. His research includes biomedical sensing for diagnostic and monitoring applications with a recent focus on the development of wearable and hand-held medical devices for underserved populations. He is a fellow of four societies (IEEE, SPIE, BMES, and AIMBE) and is an entrepreneur who has founded several start-up companies.

Biographies of the other authors are not available. 